\title{
A strong maximum principle for globally hypoelliptic operators
}

\author{
Kazuaki Taira \\ Dedicated to Professor Izumi Kubo on the occasion of his 80th birthday
}

Received: date / Accepted: date

\begin{abstract}
In this paper we study the strong maximum principle for globally hypoelliptic differential operators of second-order, and reveal the underlying analytical mechanism of propagation of maximums in terms of the Lie algebra generated by diffusion vector fields and the Fichera function. Our formulation of the strong maximum principle is coordinate-free. The results here may be applied to questions of uniqueness for degenerate elliptic boundary value problems on a manifold. Furthermore, the mechanism of propagation of maximums plays an important role in the interpretation and study of Markov processes from the viewpoint of functional analysis.
\end{abstract}

Keywords Globally hypoelliptic operator · strong maximum principle · Lie algebra $\cdot$ Fichera function

Mathematics Subject Classification (2010) 35J70 - 35H10 - 58G20 - 35K65

\section{Introduction}

The purpose of this paper is to study an intimate connection between Markov processes and partial differential equations. This will play an important role in the study of Markov processes via the theory of partial differential equations (see [31] and [32]). The present paper is an expanded and revised version of the previous works [28] through [31]. inspired by a probabilistic characterization of the strong maximum principle due to Stroock-Varadhan [27].

We begin with the following elementary result:

Let $I$ be an open interval of $\mathbf{R}$. If $u \in C^{2}(I), \frac{d^{2} u}{d x^{2}}(x) \geq 0$ in $I$

and if $u$ takes its maximum at a point of $I$, then $u$ is a constant.

Kazuaki Taira

Institute of Mathematics, University of Tsukuba, Tsukuba 305-8571, Japan

E-mail: taira@math.tsukuba.ac.jp 
This result can be extended to the $n$-dimensional case, with the operator $d^{2} / d x^{2}$ replaced by the usual Laplacian

$$
\Delta=\sum_{i=1}^{n} \frac{\partial^{2}}{\partial x_{i}^{2}}
$$

Let $\Omega$ be a connected open subset of $\mathbf{R}^{n}$. If $u \in C^{2}(\Omega), \Delta u \geq 0$ in $\Omega$ and $u$ takes its maximum at a point of $\Omega$, then $u$ is a constant.

Result (1.1) is well known by the name of the strong maximum principle for the Laplacian.

Let $L$ be a second-order, differential operator with real coefficients, defined in a connected open subset $\Omega$ of Euclidean space $\mathbf{R}^{n}$, such that

$$
L u=\sum_{i, j=1}^{n} a^{i j}(x) \frac{\partial^{2} u}{\partial x_{i} \partial x_{j}}+\sum_{i=1}^{n} b^{i}(x) \frac{\partial u}{\partial x_{i}}+c(x) u .
$$

Here:

(1) The $a^{i j}(x)$ are the components of a $C^{\infty}$ symmetric contravariant tensor of type $\left(\begin{array}{l}2 \\ 0\end{array}\right)$ on $\Omega$ and satisfy the condition

$$
\sum_{i, j=1}^{n} a^{i j}(x) \xi_{i} \xi_{j} \geq 0 \quad \text { for all } x \in \Omega \text { and all } \xi=\left(\xi_{1}, \xi_{2}, \ldots, \xi_{n}\right) \in \mathbf{R}^{n} .
$$

(2) $b^{i} \in C^{\infty}(\Omega)$ for $1 \leq i \leq n$.

(3) $c \in C^{\infty}(\Omega)$ with $L 1(x)=c(x) \leq 0$ in $\Omega$.

A strong maximum principle for the differential operator $L$ is a statement of the form:

If $u \in C^{2}(\Omega), L u \geq 0$ in $\Omega$ and if $u$ takes its greatest non-negative

value $M$ at a point $x$ of $\Omega$, then it follows that $u \equiv M$ in $\Omega$.

By using a modification of the technique originally introduced by E. Hopf [12] for elliptic operators and later adapted by L. Nirenberg [19] and A. Friedman [8] for parabolic ones, it can be proved that the maximum is propagated along a finite chain of trajectories of the diffusion vector fields

$$
\pm X_{j}= \pm \sum_{k=1}^{n} a^{j k}(x) \frac{\partial}{\partial x_{k}}, \quad 1 \leq j \leq n
$$

(see part (i) of Lemma 4.1 below).

On the other hand, Chow's theorem [5, Satz C] states that if the Lie algebra

$$
\mathcal{L}\left(X_{1}, X_{2}, \ldots, X_{n}\right)
$$

over $\mathbf{R}$ generated by the family $\left\{X_{j}\right\}_{j=1}^{n}$ of vector fields has rank $n$ at a point $x$ of $\Omega$, then there exists a neighborhood $V(x)$ of $x$ such that any point of $V(x)$ can be joined to $x$ by a finite chain of trajectories of the vector fields $\left\{ \pm X_{j}\right\}_{j=1}^{n}$. A coordinate-free definition of the Lie algebra $\mathcal{L}\left(X_{1}, X_{2}, \ldots, X_{n}\right)$ is given by the formulas (2.4) and (2.5) in the next section.

In this way, we arrive at the following well-known result (see [2, Théorème 3.1], [21, Theorem 3.1.11]): 
Theorem 1.1 If the Lie algebra $\mathcal{L}\left(X_{1}, X_{2}, \ldots, X_{n}\right)$ has rank $n$ at every point of $\Omega$, then the strong maximum principle (SMP) holds true for the operator $L$.

We give a typical example for Theorem 1.1 in the plane $\mathbf{R}^{2}(n=2)$ :

Example 1.1 Let $\Omega=\left\{\left(x_{1}, x_{2}\right):-1<x_{1}<1,-1<x_{2}<1\right\}$ (the rectangle). If we let

$$
L_{1}=\frac{\partial^{2}}{\partial x_{1}^{2}}+x_{1}^{2} \frac{\partial^{2}}{\partial x_{2}^{2}}
$$

then we have the formulas (see Remark (2.1))

$$
\begin{aligned}
& X_{1}=\frac{\partial}{\partial x_{1}}, \\
& X_{2}=x_{1}^{2} \frac{\partial}{\partial x_{2}} .
\end{aligned}
$$

It is easy to see that the vector fields

$$
\begin{aligned}
& X_{1}=\frac{\partial}{\partial x_{1}}, \\
& {\left[X_{1},\left[X_{1}, X_{2}\right]\right]=2 \frac{\partial}{\partial x_{2}}}
\end{aligned}
$$

span all vector fields at every point of $\Omega$. Namely, we have the assertion

$$
\operatorname{rank} \mathcal{L}\left(X_{1}, X_{2}\right)=2 \text { at every point of } \Omega \text {. }
$$

The purpose of this paper is to study the case where the rank of Lie algebra $\mathcal{L}\left(X_{1}, X_{2}, \ldots, X_{n}\right)$ is less than $n$ in some subset $\mathcal{M}$ of $\Omega$ under the rank condition $(\mathrm{H})$ which is formulated in Section 2. In Section 3, we impose a geometrical assumption on the exceptional set $\mathcal{M}$ (assumption (3.1)) in the rank condition $(\mathrm{H})$. Then, following Fichera [7] we introduce the Fichera function $b(x)$ by formula (3.7) (Lemmas 3.1 and 3.2). In Section 4 we give sufficient conditions for the strong maximum principle (SMP) to hold true for the operator $L$ in terms of the Lie algebra $\mathcal{L}\left(X_{1}, X_{2}, \ldots, X_{n}\right)$ and the Fichera function $b(x)$ (Theorem 4.1). In Section 5, by making use of the probabilistic representation formula (5.6) for the Dirichlet problem we give (partially) necessary conditions for the strong maximum principle (SMP) to hold true for the operator $L$ (Theorem 5.1). In Section 6 we formulate another maximum principle, called the sharp maximum principle (PMP), for the operator $L$ in terms of subunit vectors whose notion is introduced by Fefferman-Phong [6] (condition (6.1)). We give four simple examples of the strong maximum principle (SMP) via the sharp maximum principle (PMP) (Examples 6.1 through 6.4).

\section{Differential operators and Lie algebras}

In this section, we consider the case where $\Omega$ is an $n$-dimensional, $C^{\infty}$ manifold. To state a hypothesis concerning the differential operator $L$ not assumed to be written as sums of squares of vector fields, we introduce some notation and definitions. 
2.1 The Lie algebra $\mathcal{L}\left(X_{1}, X_{2}, \ldots, X_{n}\right)$

We denote by $\Gamma\left(\Omega, T^{*}(\Omega)\right)$ and by $\Gamma(\Omega, T(\Omega))$ the space of $C^{\infty}$ covector fields on $\Omega$ and the space of $C^{\infty}$ vector fields on $\Omega$, respectively.

First, we consider the space $\Gamma\left(\Omega, T(\Omega) \otimes_{S} T(\Omega)\right)$ of $C^{\infty}$ symmetric contravariant tensor fields of type $\left(\begin{array}{l}2 \\ 0\end{array}\right)$ on $\Omega$. If $x=\left(x_{1}, x_{2}, \ldots, x_{n}\right)$ is a local coordinate system in a chart on $\Omega$, we define the symmetric tensor product $\otimes_{S}$ as follows (see $[18$, Lemma 2.2.13]):

$$
\frac{\partial}{\partial x_{i}} \otimes_{S} \frac{\partial}{\partial x_{j}}=\frac{1}{2}\left(\frac{\partial}{\partial x_{i}} \otimes \frac{\partial}{\partial x_{j}}+\frac{\partial}{\partial x_{j}} \otimes \frac{\partial}{\partial x_{i}}\right) \quad \text { for } 1 \leq i, j \leq n
$$

For the leading coefficients $a^{i j}(x)$ of $L$, we let

$$
X_{j}=\sum_{k=1}^{n} a^{j k}(x) \frac{\partial}{\partial x_{k}} \quad \text { for } 1 \leq j \leq n
$$

Since we have the assertion

$$
\Phi=\sum_{i, j=1}^{n} a^{i j}(x) \frac{\partial}{\partial x_{i}} \otimes_{S} \frac{\partial}{\partial x_{j}} \in \Gamma\left(\Omega, T(\Omega) \otimes_{S} T(\Omega)\right),
$$

we can define a mapping

$$
\Psi: \Gamma\left(\Omega, T^{*}(\Omega)\right) \longrightarrow \Gamma(\Omega, T(\Omega))
$$

by the formula

$$
\Psi(\zeta)=\Phi(\zeta, \cdot) \quad \text { for every } \zeta \in \Gamma\left(\Omega, T^{*}(\Omega)\right)
$$

In terms of local coordinates $x=\left(x_{1}, x_{2}, \ldots, x_{n}\right)$ in a chart on $\Omega$, we have the formula

$$
\Gamma\left(\Omega, T^{*}(\Omega)\right) \ni \zeta=\sum_{i=1}^{n} \zeta_{i}(x) d x_{i} \longmapsto \Psi(\zeta)=\sum_{i, j=1}^{n} a^{i j}(x) \zeta_{i}(x) \frac{\partial}{\partial x_{j}} \in \Gamma(\Omega, T(\Omega)) .
$$

The proof of well-definedness of formulas (2.2) and (2.3) will be given in the next Subsection 2.2 (Lemma 2.1), due to its length.

In this way, we can associate the family $\left\{X_{j}\right\}_{j=1}^{n}$ with a family $\mathcal{Y}$ of $C^{\infty}$ vector fields on $\Omega$ defined as follows:

$$
\mathcal{Y}=\text { the image of } \Psi=\left\{\Psi(\zeta): \zeta \in \Gamma\left(\Omega, T^{*}(\Omega)\right)\right\} .
$$

In this paper, the Lie algebra $\mathcal{L}\left(X_{1}, X_{2}, \ldots, X_{n}\right)$ is meant as the Lie algebra $\mathcal{L}(\mathcal{Y})$ over $\mathbf{R}$ generated by the family $\mathcal{Y}$ :

$$
\mathcal{L}\left(X_{1}, X_{2}, \ldots, X_{n}\right)=\mathcal{L}(\mathcal{Y})
$$

It should be emphasized that this definition of the Lie algebra $\mathcal{L}\left(X_{1}, X_{2}, \ldots, X_{n}\right)$ is coordinate-free. 
Remark 2.1 If the differential operator $L$ is of the form

$$
L=\sum_{j=1}^{r} Y_{j}^{2}+Z
$$

where $Y_{1}, Y_{2}, \ldots, Y_{r}$ and $Z$ are smooth vector fields on $\Omega$, then the Lie algebra $\mathcal{L}\left(X_{1}, X_{2}, \ldots, X_{n}\right)$ may be replaced by the Lie algebra $\mathcal{L}\left(Y_{1}, Y_{2}, \ldots, Y_{r}\right)$ generated by the family $\left\{Y_{j}\right\}_{j=1}^{r}$, just as in Hörmander [14] and Bony [2] (see [31, the proof of Theorem 7.2.4]).

In this paper, we let

$$
\mathcal{M}=\left\{x \in \Omega \text { : the rank of } \mathcal{L}\left(X_{1}, X_{2}, \ldots, X_{n}\right) \text { at } x \text { is less than } n\right\},
$$

and impose the following rank condition on the Lie algebra $\mathcal{L}\left(X_{1}, X_{2}, \ldots, X_{n}\right)$ defined by formulas (2.4) and (2.5):

(H) The exceptional set $\mathcal{M}$ is a closed hypersurface in $\Omega$ such that

$$
\operatorname{rank} \mathcal{L}\left(X_{1}, X_{2}, \ldots, X_{n}\right)=n \quad \text { outside } \mathcal{M}
$$

2.2 The well-definedness of formulas (2.2) and (2.3)

Now we are in a position to prove the well-definedness of the mapping $\Psi$ defined by formulas (2.2) and (2.3):

Lemma 2.1 Let $x_{0}$ be an arbitrary point of $\Omega$ and consider, in a neighborhood $U$ of $x_{0}, a C^{2}$-diffeomorphism

$$
y=F(x)=\left(F_{1}(x), F_{2}(x), \ldots, F_{n}(x)\right) \quad \text { for } x \in U .
$$

Assume that

$$
\zeta=\sum_{i=1}^{n} \zeta_{i}(x) d x_{i}=\sum_{\ell=1}^{n} \eta_{\ell}(y) d y_{\ell} \in \Gamma\left(\Omega, T^{*}(\Omega)\right) .
$$

Then we have, by formulas (2.6) and (2.7),

$$
\begin{aligned}
\Psi(\zeta) & =\sum_{j=1}^{n}\left(\sum_{i=1}^{n} a^{i j}(x) \zeta_{i}(x)\right) \frac{\partial}{\partial x_{j}} \\
& =\sum_{m=1}^{n}\left(\sum_{\ell=1}^{n} \widetilde{a}^{\ell m}(y) \eta_{\ell}(y)\right) \frac{\partial}{\partial y_{m}} \in \Gamma(\Omega, T(\Omega)),
\end{aligned}
$$

where the $\widetilde{a}^{\ell m}(y)$ are the components of a $C^{\infty}$ symmetric contravariant tensor of type $\left(\begin{array}{l}2 \\ 0\end{array}\right)$ defined by the formulas

$$
\begin{aligned}
\widetilde{a}^{\ell m}(y) & =\sum_{i, j=1}^{n} a^{i j}\left(F^{-1}(y)\right) \frac{\partial y_{\ell}}{\partial x_{i}} \frac{\partial y_{m}}{\partial x_{j}} \\
& =\sum_{i, j=1}^{n} a^{i j}\left(F^{-1}(y)\right) \frac{\partial F_{\ell}}{\partial x_{i}} \frac{\partial F_{m}}{\partial x_{j}} \quad \text { for } 1 \leq \ell, m \leq n .
\end{aligned}
$$


Proof First, since we have, by formula (2.6),

$$
\begin{aligned}
\frac{\partial}{\partial x_{i}} & =\sum_{\ell=1}^{n} \frac{\partial y_{\ell}}{\partial x_{i}} \frac{\partial}{\partial y_{\ell}}=\sum_{\ell=1}^{n} \frac{\partial F_{\ell}}{\partial x_{i}} \frac{\partial}{\partial y_{\ell}} \quad \text { for } 1 \leq i \leq n . \\
\frac{\partial}{\partial x_{j}} & =\sum_{m=1}^{n} \frac{\partial y_{m}}{\partial x_{j}} \frac{\partial}{\partial y_{m}}=\sum_{m=1}^{n} \frac{\partial F_{m}}{\partial x_{j}} \frac{\partial}{\partial y_{m}} \quad \text { for } 1 \leq j \leq n,
\end{aligned}
$$

we obtain from formula (2.1) that

$$
\begin{aligned}
& \frac{\partial}{\partial x_{i}} \otimes_{S} \frac{\partial}{\partial x_{j}}=\frac{1}{2}\left(\frac{\partial}{\partial x_{i}} \otimes \frac{\partial}{\partial x_{j}}+\frac{\partial}{\partial x_{j}} \otimes \frac{\partial}{\partial x_{i}}\right) \\
= & \frac{1}{2}\left(\sum_{\ell=1}^{n} \frac{\partial y_{\ell}}{\partial x_{i}} \frac{\partial}{\partial y_{\ell}} \otimes \sum_{m=1}^{n} \frac{\partial y_{m}}{\partial x_{j}} \frac{\partial}{\partial y_{m}}+\sum_{m=1}^{n} \frac{\partial y_{m}}{\partial x_{j}} \frac{\partial}{\partial y_{m}} \otimes \sum_{\ell=1}^{n} \frac{\partial y_{\ell}}{\partial x_{i}} \frac{\partial}{\partial y_{\ell}}\right) \\
= & \sum_{\ell, m=1}^{n} \frac{\partial y_{\ell}}{\partial x_{i}} \frac{\partial y_{m}}{\partial x_{j}} \cdot \frac{1}{2}\left(\frac{\partial}{\partial y_{\ell}} \otimes \frac{\partial}{\partial y_{m}}+\frac{\partial}{\partial y_{m}} \otimes \frac{\partial}{\partial y_{\ell}}\right) \\
= & \sum_{\ell, m=1}^{n} \frac{\partial y_{\ell}}{\partial x_{i}} \frac{\partial y_{m}}{\partial x_{j}}\left(\frac{\partial}{\partial y_{\ell}} \otimes_{S} \frac{\partial}{\partial y_{m}}\right) .
\end{aligned}
$$

Hence we have the formula

$$
\begin{aligned}
\Phi & =\sum_{i, j=1}^{n} a^{i j}(x) \frac{\partial}{\partial x_{i}} \otimes_{S} \frac{\partial}{\partial x_{j}} \\
& =\sum_{i, j=1}^{n} a^{i j}(x)\left(\sum_{\ell, m=1}^{n} \frac{\partial y_{\ell}}{\partial x_{i}} \frac{\partial y_{m}}{\partial x_{j}}\left(\frac{\partial}{\partial y_{\ell}} \otimes_{S} \frac{\partial}{\partial y_{m}}\right)\right) \\
& =\sum_{\ell, m=1}^{n}\left(\sum_{i, j=1}^{n} a^{i j}\left(F^{-1}(y)\right) \frac{\partial F_{\ell}}{\partial x_{i}} \frac{\partial F_{m}}{\partial x_{j}}\right) \frac{\partial}{\partial y_{\ell}} \otimes_{S} \frac{\partial}{\partial y_{m}} \\
& =\sum_{\ell, m=1}^{n} \tilde{a}^{\ell m}(y) \frac{\partial}{\partial y_{\ell}} \otimes_{S} \frac{\partial}{\partial y_{m}} .
\end{aligned}
$$

This proves the desired assertion (2.2).

Secondly, by formula (2.7) it follows that

$$
\zeta=\sum_{\ell=1}^{n} \eta_{\ell}(y) d y_{\ell}=\sum_{\ell=1}^{n} \eta_{\ell}(y)\left(\sum_{i=1}^{n} \frac{\partial y_{\ell}}{\partial x_{i}} d x_{i}\right)=\sum_{i=1}^{n}\left(\sum_{\ell=1}^{n} \frac{\partial y_{\ell}}{\partial x_{i}} \eta_{\ell}(y)\right) d x_{i}
$$

so that

$$
\zeta_{i}(x)=\sum_{\ell=1}^{n} \frac{\partial y_{\ell}}{\partial x_{i}} \eta_{\ell}(y)=\sum_{\ell=1}^{n} \frac{\partial F_{\ell}}{\partial x_{i}} \eta_{\ell}(y) \quad \text { for } 1 \leq i \leq n .
$$

Therefore, by using formulas (2.10b) and (2.11) we obtain that

$$
\sum_{i, j=1}^{n} a^{i j}(x) \zeta_{i}(x) \frac{\partial}{\partial x_{j}}=\sum_{i, j=1}^{n} a^{i j}(x)\left(\sum_{\ell=1}^{n} \frac{\partial y_{\ell}}{\partial x_{i}} \eta_{\ell}(y)\right)\left(\sum_{m=1}^{n} \frac{\partial y_{m}}{\partial x_{j}} \frac{\partial}{\partial y_{m}}\right)
$$




$$
\begin{aligned}
& =\sum_{\ell, m=1}^{n}\left(\sum_{i, j=1}^{n} a^{i j}\left(F^{-1}(y)\right) \frac{\partial y_{\ell}}{\partial x_{i}} \frac{\partial y_{m}}{\partial x_{j}}\right) \eta_{\ell}(y) \frac{\partial}{\partial y_{m}} \\
& =\sum_{\ell, m=1}^{n} \widetilde{a}^{\ell m}(y) \eta_{\ell}(y) \frac{\partial}{\partial y_{m}} .
\end{aligned}
$$

This proves the desired assertion (2.8).

The proof of Lemma 2.1 is complete.

\section{The Fichera function}

In this section, we impose the following geometrical assumption on the exceptional set $\mathcal{M}$ in the rank condition (H) (see Figure 3.1):

The set $\mathcal{M}$ is a closed hypersurface defined locally by the equation

$$
\mathcal{M}=\{x \in \Omega: \varphi(x)=0\} \text { with } \operatorname{grad} \varphi \neq 0 .
$$

Here

$$
\boldsymbol{\nu}=\left(\nu_{1}, \nu_{2}, \ldots, \nu_{n}\right)=\operatorname{grad} \varphi
$$

is the normal to the hypersurface $\mathcal{M}$.

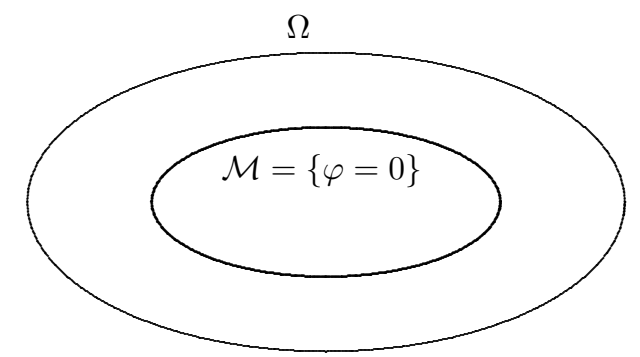

Fig. 3.1 The closed hypersurface $\mathcal{M}$ in $\Omega$

First, we introduce two disjoint subsets $\Sigma_{3}$ and $\Sigma^{0}$ of $\mathcal{M}$ respectively by the formulas

$$
\begin{aligned}
& \Sigma_{3}=\left\{x \in \mathcal{M}: \sum_{i, j=1}^{n} a^{i j}(x) \nu_{i} \nu_{j}>0\right\}, \\
& \Sigma^{0}=\left\{x \in \mathcal{M}: \sum_{i, j=1}^{n} a^{i j}(x) \nu_{i} \nu_{j}=0\right\}=\mathcal{M} \backslash \Sigma_{3} .
\end{aligned}
$$

The next lemma justifies the definition of the sets $\Sigma_{3}$ and $\Sigma^{0}$ (cf. [21, Theorem 1.1.1]):

Lemma 3.1 The sets $\Sigma_{3}$ and $\Sigma^{0}$ are invariant under $C^{2}$-diffeomorphisms preserving normal vectors to the set $\mathcal{M}$. 
Proof Let $x_{0}$ be an arbitrary point of $\mathcal{M}$ and consider, in a neighborhood $U$ of $x_{0}$, a $C^{2}$-diffeomorphism

$$
y=F(x)=\left(F_{1}(x), F_{2}(x), \ldots, F_{n}(x)\right), \quad x \in U,
$$

which preserves the normal vector $\boldsymbol{\nu}=\left(\nu_{1}, \nu_{2}, \ldots, \nu_{n}\right)$ to $\mathcal{M}$. Then we have, by assumption (3.1),

$$
\mathcal{M} \cap U=\{y \in U: \Phi(y)=0\}, \quad \Phi=\varphi \circ F^{-1},
$$

and

$$
\nu_{i}=\frac{\partial \varphi}{\partial x_{i}}=\sum_{\ell=1}^{n} \frac{\partial \Phi}{\partial y_{\ell}} \frac{\partial y_{\ell}}{\partial x_{i}}=\sum_{\ell=1}^{n} \frac{\partial \Phi}{\partial y_{\ell}} \frac{\partial F_{\ell}}{\partial x_{i}} \quad \text { for } 1 \leq i \leq n
$$

Furthermore, we can rewrite the differential operator $L$ in the form

$$
\begin{aligned}
L= & \sum_{i, j=1}^{n} a^{i j}(x) \frac{\partial^{2}}{\partial x_{i} \partial x_{j}}+\sum_{i=1}^{n} b^{i}(x) \frac{\partial}{\partial x_{i}}+c(x) \\
= & \sum_{\ell, m=1}^{n}\left(\sum_{i, j=1}^{n} a^{i j}\left(F^{-1}(y)\right) \frac{\partial F_{\ell}}{\partial x_{i}} \frac{\partial F_{m}}{\partial x_{j}}\right) \frac{\partial^{2}}{\partial y_{\ell} \partial y_{m}} \\
& +\sum_{\ell=1}^{n}\left(\sum_{i=1}^{n} b^{i}\left(F^{-1}(y)\right) \frac{\partial F_{\ell}}{\partial x_{i}}+\sum_{i, j=1}^{n} a^{i j}\left(F^{-1}(y)\right) \frac{\partial^{2} F_{\ell}}{\partial x_{i} \partial x_{j}}\right) \frac{\partial}{\partial y_{\ell}} \\
& +c\left(F^{-1}(y)\right) \\
:= & \sum_{\ell, m=1}^{n} \tilde{a}^{\ell m}(y) \frac{\partial^{2}}{\partial y_{\ell} \partial y_{m}}+\sum_{\ell=1}^{n} \widetilde{b}^{\ell}(y) \frac{\partial}{\partial y_{\ell}}+\widetilde{c}(y) .
\end{aligned}
$$

However, we have, by formulas (3.4) and (2.9),

$$
\begin{aligned}
\sum_{i, j=1}^{n} a^{i j}(x) \frac{\partial \varphi}{\partial x_{i}} \frac{\partial \varphi}{\partial x_{j}} & =\sum_{i, j=1}^{n} a^{i j}(x)\left(\sum_{\ell, m=1}^{n} \frac{\partial \Phi}{\partial y_{\ell}} \frac{\partial y_{\ell}}{\partial x_{i}} \frac{\partial \Phi}{\partial y_{m}} \frac{\partial y_{m}}{\partial x_{j}}\right) \\
& =\sum_{\ell, m=1}^{n}\left(\sum_{i, j=1}^{n} a^{i j}\left(F^{-1}(y)\right) \frac{\partial y_{\ell}}{\partial x_{i}} \frac{\partial y_{m}}{\partial x_{j}}\right) \frac{\partial \Phi}{\partial y_{\ell}} \frac{\partial \Phi}{\partial y_{m}} \\
& =\sum_{\ell, m=1}^{n} \tilde{a}^{\ell m}(y) \frac{\partial \Phi}{\partial y_{\ell}} \frac{\partial \Phi}{\partial y_{m}} .
\end{aligned}
$$

This proves the invariance of the sets $\Sigma_{3}$ and $\Sigma^{0}$, since the diffeomorphism $F$ preserves normal vectors and so grad $\Phi$ has the same direction as the inward normal $\boldsymbol{\nu}$. Indeed, we obtain from formula (3.6) that

$$
\begin{aligned}
\sum_{i, j=1}^{n} a^{i j}(x) \frac{\partial \varphi}{\partial x_{i}} \frac{\partial \varphi}{\partial x_{j}}>0 \Longleftrightarrow \sum_{\ell, m=1}^{n} \tilde{a}^{\ell m}(y) \frac{\partial \Phi}{\partial y_{\ell}} \frac{\partial \Phi}{\partial y_{m}}>0 \\
\sum_{i, j=1}^{n} a^{i j}(x) \frac{\partial \varphi}{\partial x_{i}} \frac{\partial \varphi}{\partial x_{j}}=0 \Longleftrightarrow \sum_{\ell, m=1}^{n} \tilde{a}^{\ell m}(y) \frac{\partial \Phi}{\partial y_{\ell}} \frac{\partial \Phi}{\partial y_{m}}=0 .
\end{aligned}
$$

The proof of Lemma 3.1 is complete. 
Following Fichera [7], we introduce a function $b(x)$, called the Fichera function, defined by the formula

$$
b(x)=\sum_{i=1}^{n}\left(b^{i}(x)-\sum_{j=1}^{n} \frac{\partial a^{i j}}{\partial x_{j}}(x)\right) \nu_{i}=\sum_{i=1}^{n}\left(b^{i}(x)-\sum_{j=1}^{n} \frac{\partial a^{i j}}{\partial x_{j}}(x)\right) \frac{\partial \varphi}{\partial x_{i}} .
$$

The next lemma justifies the definition (3.7) of the Fichera function $b(x)$ on the set $\Sigma^{0}$ (cf. [21, Lemma 1.1.1]):

Lemma 3.2 The Fichera function $b(x)$ defined on the set $\Sigma^{0}$ is invariant under $C^{2}$ diffeomorphisms preserving normal vectors to the set $\mathcal{M}$.

Proof Let $x_{0}$ be an arbitrary point of $\mathcal{M}$ and consider, in a neighborhood $U$ of $x_{0}$, a $C^{2}$-diffeomorphism

$$
y=F(x)=\left(F_{1}(x), F_{2}(x), \ldots, F_{n}(x)\right),
$$

as in the proof of Lemma 3.1 .

Then we have, by formulas (3.5) and (3.4),

$$
\begin{aligned}
\widetilde{b}(y) & =\sum_{\ell=1}^{n}\left(\widetilde{b}^{\ell}(y)-\sum_{m=1}^{n} \frac{\partial \widetilde{a}^{\ell m}}{\partial y_{m}}(y)\right) \frac{\partial \Phi}{\partial y_{\ell}} \\
& =\sum_{\ell=1}^{n}\left(\sum_{i=1}^{n} b^{i}(x) \frac{\partial F_{\ell}}{\partial x_{i}}+\sum_{i, j=1}^{n} a^{i j}(x) \frac{\partial^{2} F_{\ell}}{\partial x_{i} \partial x_{j}}\right) \frac{\partial \Phi}{\partial y_{\ell}}-\sum_{\ell=1}^{n} \sum_{m=1}^{n} \frac{\partial \widetilde{a}^{\ell m}}{\partial y_{m}}(y) \frac{\partial \Phi}{\partial y_{\ell}} \\
& =\sum_{i=1}^{n} b^{i}(x) \frac{\partial \varphi}{\partial x_{i}}+\sum_{\ell=1}^{n}\left(\sum_{i, j=1}^{n} a^{i j}(x) \frac{\partial^{2} F_{\ell}}{\partial x_{i} \partial x_{j}}\right) \frac{\partial \Phi}{\partial y_{\ell}}-\sum_{\ell=1}^{n} \sum_{m=1}^{n} \frac{\partial \widetilde{a}^{\ell m}}{\partial y_{m}}(y) \frac{\partial \Phi}{\partial y_{\ell}} .
\end{aligned}
$$

Moreover, we can calculate the last term in formula (3.8) as follows:

$$
\begin{aligned}
& \sum_{\ell=1}^{n} \sum_{m=1}^{n} \frac{\partial \widetilde{a}^{\ell m}}{\partial y_{m}}(y) \frac{\partial \Phi}{\partial y_{\ell}} \\
= & \sum_{\ell=1}^{n} \sum_{m=1}^{n} \frac{\partial}{\partial y_{m}}\left(\sum_{i, j=1}^{n} a^{i j}(x) \frac{\partial F_{\ell}}{\partial x_{i}} \frac{\partial F_{m}}{\partial x_{j}}\right) \frac{\partial \Phi}{\partial y_{\ell}} \\
= & \sum_{m=1}^{n} \sum_{i, j=1}^{n} \frac{\partial a^{i j}}{\partial y_{m}} \frac{\partial F_{m}}{\partial x_{j}} \frac{\partial \varphi}{\partial x_{i}}+\sum_{\ell=1}^{n} \sum_{i, j=1}^{n} a^{i j}(x) \frac{\partial^{2} F_{\ell}}{\partial x_{i} \partial x_{j}} \frac{\partial \Phi}{\partial y_{\ell}} \\
& +\sum_{i, j=1}^{n} a^{i j}(x) \frac{\partial \varphi}{\partial x_{i}} \sum_{m=1}^{n} \frac{\partial^{2} F_{m}}{\partial y_{m} \partial x_{j}},
\end{aligned}
$$

since we have, by formulas (2.10b),

$$
\sum_{m=1}^{n} \frac{\partial^{2} F_{\ell}}{\partial y_{m} \partial x_{i}} \frac{\partial F_{m}}{\partial x_{j}}=\sum_{m=1}^{n} \frac{\partial F_{m}}{\partial x_{j}} \frac{\partial}{\partial y_{m}}\left(\frac{\partial F_{\ell}}{\partial x_{i}}\right)=\frac{\partial^{2} F_{\ell}}{\partial x_{i} \partial x_{j}} .
$$

Therefore, by combining formulas (3.8) and (3.9) we obtain that

$$
\tilde{b}(y)=\sum_{\ell=1}^{n}\left(\tilde{b}^{\ell}(y)-\sum_{m=1}^{n} \frac{\partial \widetilde{a}^{\ell m}}{\partial y_{m}}(y)\right) \frac{\partial \Phi}{\partial y_{\ell}}
$$




$$
\begin{aligned}
& =\sum_{i=1}^{n} b^{i}(x) \frac{\partial \varphi}{\partial x_{i}}+\sum_{\ell=1}^{n}\left(\sum_{i, j=1}^{n} a^{i j}(x) \frac{\partial^{2} F_{\ell}}{\partial x_{i} \partial x_{j}}\right) \frac{\partial \Phi}{\partial y_{\ell}}-\sum_{\ell=1}^{n} \sum_{m=1}^{n} \frac{\partial \widetilde{a}^{\ell m}}{\partial y_{m}}(y) \frac{\partial \Phi}{\partial y_{\ell}} \\
& =\sum_{i=1}^{n} b^{i}(x) \frac{\partial \varphi}{\partial x_{i}}-\sum_{i, j=1}^{n}\left(\sum_{m=1}^{n} \frac{\partial a^{i j}}{\partial y_{m}} \frac{\partial F_{m}}{\partial x_{j}}\right) \frac{\partial \varphi}{\partial x_{i}}-\sum_{i, j=1}^{n} a^{i j}(x) \frac{\partial \varphi}{\partial x_{i}} \sum_{m=1}^{n} \frac{\partial^{2} F_{m}}{\partial y_{m} \partial x_{j}} \\
& =\sum_{i=1}^{n} b^{i}(x) \frac{\partial \varphi}{\partial x_{i}}-\sum_{i=1}^{n}\left(\sum_{j=1}^{n} \frac{\partial a^{i j}}{\partial x_{j}}\right) \frac{\partial \varphi}{\partial x_{i}}-\sum_{j=1}^{n}\left(\sum_{i=1}^{n} a^{i j}(x) \frac{\partial \varphi}{\partial x_{i}}\right) \sum_{m=1}^{n} \frac{\partial^{2} F_{m}}{\partial y_{m} \partial x_{j}} \\
& =\sum_{i=1}^{n}\left(b^{i}(x)-\sum_{j=1}^{n} \frac{\partial a^{i j}}{\partial x_{j}}\right) \frac{\partial \varphi}{\partial x_{i}}-\sum_{j=1}^{n}\left(\sum_{i=1}^{n} a^{i j}(x) \frac{\partial \varphi}{\partial x_{i}}\right) \sum_{m=1}^{n} \frac{\partial^{2} F_{m}}{\partial y_{m} \partial x_{j}} \\
& =b(x)-\sum_{j=1}^{n}\left(\sum_{i=1}^{n} a^{i j}(x) \frac{\partial \varphi}{\partial x_{i}}\right) \sum_{m=1}^{n} \frac{\partial^{2} F_{m}}{\partial y_{m} \partial x_{j}} .
\end{aligned}
$$

On the other hand, by applying Oleĭnik-Radkevič [21, Corollary 1 to Lemma 1.7.1] we have the inequality

$$
\left|\sum_{i=1}^{n} a^{i j}(x) \frac{\partial \varphi}{\partial x_{i}}\right|^{2} \leq 2 a^{j j}(x) \sum_{i, k=1}^{n} a^{i k}(x) \frac{\partial \varphi}{\partial x_{i}} \frac{\partial \varphi}{\partial x_{k}} \quad \text { for } 1 \leq j \leq n .
$$

Hence, on the set

$$
\Sigma^{0}=\left\{x \in \mathcal{M}: \sum_{i, k=1}^{n} a^{i k}(x) \nu_{i} \nu_{k}=0\right\}
$$

it follows that

$$
\sum_{i=1}^{n} a^{i j}(x) \frac{\partial \varphi}{\partial x_{i}}=0 \quad \text { for } 1 \leq j \leq n .
$$

Namely, we find that the last term in the right-hand side of formula (3.10) vanishes on the set $\Sigma^{0}$ :

$$
\sum_{j=1}^{n}\left(\sum_{i=1}^{n} a^{i j}(x) \frac{\partial \varphi}{\partial x_{i}}\right) \sum_{m=1}^{n} \frac{\partial^{2} F_{m}}{\partial y_{m} \partial x_{j}}=0 \quad \text { on } \Sigma^{0} .
$$

In this way, we obtain from assertion (3.11) and formula (3.10) that

$$
b(x)=\widetilde{b}(y) \quad \text { on } \Sigma^{0} .
$$

This proves the invariance of the Fichera function $b(x)$ on the set $\Sigma^{0}$.

The proof of Lemma 3.2 is complete. 


\section{Sufficient conditions}

Our first main result is stated as follows (cf. [21, Theorem 3.1.13]):

Theorem 4.1 (Sufficiency) Assume that the exceptional set $\mathcal{M}$ is a closed, connected hypersurface such that the complement $\Omega \backslash \mathcal{M}$ has two connected components. Furthermore, we make the following assumption:

(A) The function

$$
\sum_{i, j=1}^{n} a^{i j}\left(x^{\prime}\right) \nu_{i} \nu_{j}+\left|b\left(x^{\prime}\right)\right|
$$

does not vanish identically on the hypersurface $\mathcal{M}$ and, for each point $x^{\prime}$ of the set

$$
\mathcal{N}=\left\{x^{\prime} \in \mathcal{M}: \sum_{i, j=1}^{n} a^{i j}\left(x^{\prime}\right) \nu_{i} \nu_{j}+\left|b\left(x^{\prime}\right)\right|=0\right\},
$$

there exists a point $x_{0}^{\prime}$ of $\mathcal{M} \backslash \mathcal{N}$ such that $x^{\prime}$ and $x_{0}^{\prime}$ are connected by a finite chain of trajectories of the vector fields $\left\{ \pm X_{j}\right\}_{j=1}^{n}$ (see Figure 4.1).

Then the strong maximum principle (SMP) holds true for the operator $L$ if and only if one of the following two conditions (B) and (C) is satisfied (see Figure 4.2):

(B) The function

$$
\sum_{i, j=1}^{n} a^{i j}\left(x^{\prime}\right) \nu_{i} \nu_{j}
$$

does not vanish identically on the hypersurface $\mathcal{M}$.

(C) If the function

$$
\sum_{i, j=1}^{n} a^{i j}\left(x^{\prime}\right) \nu_{i} \nu_{j}
$$

vanishes identically on the hypersurface $\mathcal{M}$, then there exist at least two points $x_{+}^{\prime}$ and $x_{-}^{\prime}$ of $\mathcal{M}$ such that

$$
b\left(x_{ \pm}^{\prime}\right)=\sum_{i=1}^{n}\left(b^{i}\left(x_{ \pm}^{\prime}\right)-\sum_{j=1}^{n} \frac{\partial a^{i j}}{\partial x_{j}}\left(x_{ \pm}^{\prime}\right)\right) \nu_{i} \gtrless 0 .
$$

Remark 4.1 The assumption (A) implies that the maximum starting at $\mathcal{N}$ (where the maximum may stay) goes out of $\mathcal{N}$. On the other hand, the maximum can traverse $\mathcal{M}$ from every side of $\mathcal{M}$ if and only if one of the conditions (B) and (C) is satisfied.

We give two simple examples for Theorem 4.1 in the plane $\mathbf{R}^{2}(n=2)$ :

Example 4.1 Let $\Omega=\left\{\left(x_{1}, x_{2}\right):-1<x_{1}<1,-1<x_{2}<1\right\}$ (the rectangle). We let

$$
L_{2}=\frac{\partial^{2}}{\partial x_{1}^{2}}+\exp \left[-\frac{1}{x_{1}^{2}}\right] \frac{\partial^{2}}{\partial x_{2}^{2}} .
$$

Then we have the assertions

$$
\mathcal{M}=\left\{\left(0, x_{2}\right):-1<x_{2}<1\right\}=\{0\} \times(-1,1),
$$




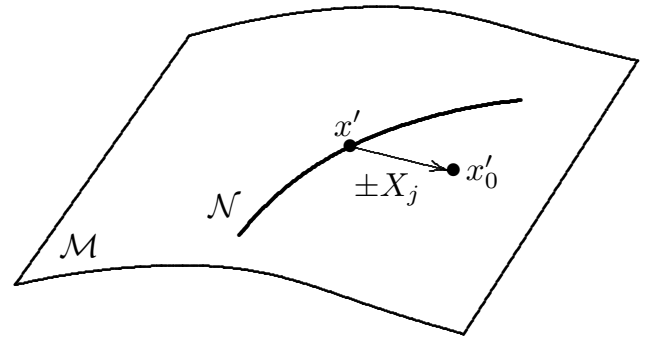

Fig. 4.1 The condition (A)

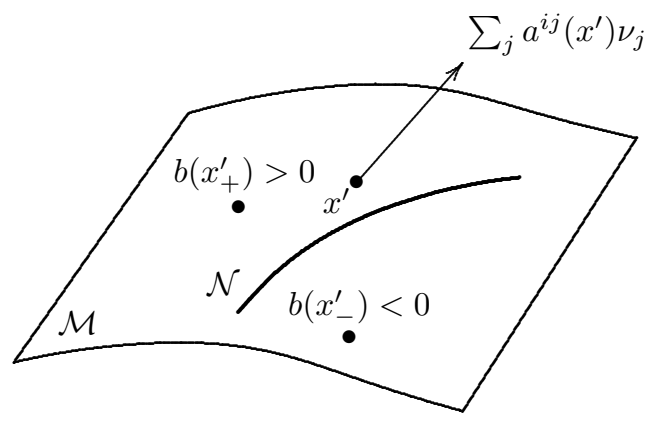

Fig. 4.2 The conditions (B) and (C)

$$
\mathcal{N}=\emptyset
$$

It is easy to see that the conditions (A) and (B) hold true. Indeed, it suffices to note that

$$
\begin{cases}\sum_{i, j=1}^{2} a^{i j}\left(x_{1}, x_{2}\right) \nu_{i} \nu_{j} \equiv 1 & \text { on } \mathcal{M} \\ b\left(x_{1}, x_{2}\right) \equiv 0 & \text { on } \mathcal{M}\end{cases}
$$

Example 4.2 Let $\Omega=\left\{\left(x_{1}, x_{2}\right):-1<x_{1}<1,-1<x_{2}<1\right\}$ (the rectangle). We let

$$
L_{3}=\exp \left[-\frac{1}{x_{1}^{2}}\right] \frac{\partial^{2}}{\partial x_{1}^{2}}+\frac{\partial^{2}}{\partial x_{2}^{2}}+x_{2} \frac{\partial}{\partial x_{1}} .
$$

Then we have the assertions

$$
\begin{aligned}
& \mathcal{M}=\left\{\left(0, x_{2}\right):-1<x_{2}<1\right\}=\{0\} \times(-1,1), \\
& \mathcal{N}=\{(0,0)\}
\end{aligned}
$$

It is easy to see that the conditions (A) and (C) hold true. Indeed, it suffices to note that

$$
\begin{cases}\sum_{i, j=1}^{2} a^{i j}\left(x_{1}, x_{2}\right) \nu_{i} \nu_{j} \equiv 0 & \text { on } \mathcal{M} \\ b\left(x_{1}, x_{2}\right)=x_{2} & \text { on } \mathcal{M} .\end{cases}
$$


4.1 Proof of Theorem 4.1

Assume that

$$
\left\{\begin{array}{l}
u \in C^{2}(\Omega) \\
L u \geq 0 \quad \text { in } \Omega \\
M=\sup _{x \in \Omega} u(x) \geq 0
\end{array}\right.
$$

and let

$$
F=\{x \in \Omega: u(x)=M\} .
$$

Our proof is essentially based on the following lemma (cf. [1], [2], [11], [21], [24]):

Lemma 4.1 (i) Let $x(t)$ be a finite chain of trajectories of the diffusion vector fields

$$
\pm X_{j}= \pm \sum_{k=1}^{n} a^{j k}(x) \frac{\partial}{\partial x_{k}} \quad \text { for } 1 \leq j \leq n .
$$

If we have the assertion

$$
x\left(t_{0}\right) \in F \quad \text { for some } t_{0},
$$

then it follows that

$$
x(t) \in F \quad \text { for all } t \geq t_{0} .
$$

(ii) Let $x_{0}$ be a point of $\mathcal{M}$ such that

$$
\sum_{i, j=1}^{n} a^{i j}\left(x_{0}\right) \nu_{i} \nu_{j}=0 \quad \text { and } L \varphi\left(x_{0}\right)>0 .
$$

If $x_{0} \in F$, then the connected component

$$
\{x \in \Omega: \varphi(x)>0\}
$$

of $\Omega \backslash \mathcal{M}$ is contained in the set $F$

Similarly, let $x_{0}$ be a point of $\mathcal{M}$ such that

$$
\sum_{i, j=1}^{n} a^{i j}\left(x_{0}\right) \nu_{i} \nu_{j}=0 \quad \text { and } L \varphi\left(x_{0}\right)<0 .
$$

If $x_{0} \in F$, then the connected component

$$
\{x \in \Omega: \varphi(x)<0\}
$$

of $\Omega \backslash \mathcal{M}$ is contained in the set $F$.

Proof (i) In order to prove part (i), it suffices to note that [2, Proposition 3.1] remains valid for the operator $L$ of the form (1.2), as is proved by Oleĭnik-Radkevič [21, Theorem 3.1.6]. More precisely, the reader might refer to Redheffer [24, Lemma 7] and Amano [1, Lemma 3].

(ii) Without loss of generality, we may choose a local coordinate system

$$
y=\left(y^{\prime}, y_{n}\right)=\left(y_{1}, \ldots, y_{n-1}, y_{n}\right)
$$

in a neighborhood $U$ of $x_{0}$ such that (see Figure 4.3) 
(1) $x_{0}=$ the origin, $\varphi(y)=y_{n}$ and $\boldsymbol{\nu}=(0, \ldots, 0,1)$;

(2) $U$ is a unit ball about the origin;

(3) $L$ is of the form

$$
L=\sum_{i, j=1}^{n} \alpha^{i j}(y) \frac{\partial^{2}}{\partial y_{i} \partial y_{j}}+\sum_{i=1}^{n} \beta^{i}(y) \frac{\partial}{\partial y_{i}}+c(y) .
$$

Here we remark that

$$
\alpha^{n n}(0)=0,
$$

since we have the condition

$$
\sum_{i, j=1}^{n} a^{i j}\left(x_{0}\right) \nu_{i} \nu_{j}=0
$$

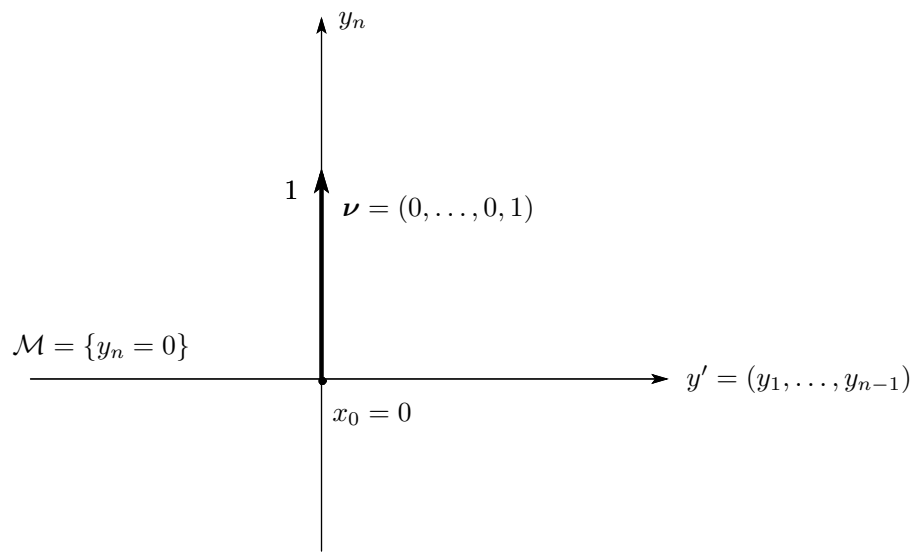

Fig. 4.3 The local coordinate system $y=\left(y^{\prime}, y_{n}\right)=\left(y_{1}, \ldots, y_{n-1}, y_{n}\right)$ near $x_{0}=0$

By replacing $y_{n}$ by $-y_{n}$ if necessary, it suffices to consider the case where

$$
L \varphi\left(x_{0}\right)=\beta^{n}(0)>0 .
$$

Our proof of part (ii) is based on a reduction to absurdity. We assume, to the contrary, that the assertion does not hold true. Then it follows from part (i) and Chow's theorem [5, Satz C] that there is no point of $F$ in the upper half-space $\left\{y_{n}>0\right\}$. We let

$$
h(y)=\varepsilon\left(y_{1}^{2}+y_{2}^{2}+\ldots+y_{n-1}^{2}\right)+\left(y_{n}-1\right)^{2},
$$

where $0<\varepsilon<1$ will be chosen later on. We remark that the ellipsoid

$$
\left\{y \in \mathbf{R}^{n}: h(y) \leq 1\right\}=\{h(y) \leq 1\}
$$

touches the set $F$ only at the origin. 
Consider the function

$$
v(y)=\exp [1-h(y)]-1 .
$$

Since $\alpha^{n n}(0)=0$, we have the formula

$$
L v(0)=-2 \varepsilon \sum_{i=1}^{n-1} \alpha^{i i}(0)+2 \beta^{n}(0) .
$$

By condition (4.1), we can choose $\varepsilon$ so small that

$$
L v>0 \text { in some neighborhood } V \text { of the origin. }
$$

If $\lambda$ is a positive constant, we let

$$
w(y):=u(y)+\lambda v(y) .
$$

Since $L u \geq 0$ in $\Omega$, it follows from assertion (4.2) that

$$
L w>0 \text { in } V \text {. }
$$

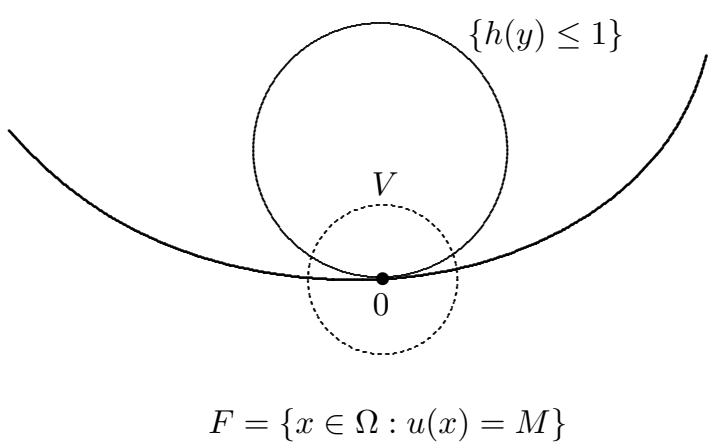

Fig. 4.4 The closed set $F$ and the neighborhood $V$ of $x_{0}=0$

We remark that the distance between the set $\partial V \cap F$ and the set

$$
\{v(y) \geq 0\}=\{\exp [1-h(y)] \geq 1\}=\{h(y) \leq 1\}
$$

is positive, where $\partial V$ is the boundary of $V$ (see Figure 4.4). Hence we can choose the constant $\lambda$ so small that

$$
w(y)<M \quad \text { on } \partial V .
$$

However, since $w(0)=u(0)=M$, it follows that the function $w(y)$ takes its greatest non-negative value inside $V$. Therefore, we have the inequality

$$
\begin{aligned}
L w(0) & =\sum_{i, j=1}^{n} \alpha^{i j}(0) \frac{\partial^{2} w}{\partial x_{i} \partial x_{j}}(0)+\sum_{i=1}^{n} \beta^{i}(0) \frac{\partial w}{\partial x_{i}}(0)+c(0) w(0) \\
& =\sum_{i, j=1}^{n} \alpha^{i j}(0) \frac{\partial^{2} w}{\partial x_{i} \partial x_{j}}(0)+c(0) w(0) \leq \sum_{i, j=1}^{n} \alpha^{i j}(0) \frac{\partial^{2} w}{\partial x_{i} \partial x_{j}}(0) \\
& \leq 0 .
\end{aligned}
$$

This contradicts inequality (4.3).

The proof of lemma 4.1 is complete. 
4.2 End of Proof of Theorem 4.1

The proof of Theorem 4.1 is divided into two steps.

Step 1: First, we have the following formula for the Fichera function $b(x)$ on the set $\mathcal{M}$ (cf. [27, the proof of Lemma 7.1]):

Claim 4.1 If the function

$$
\sum_{i, j=1}^{n} a^{i j}(x) \nu_{i} \nu_{j}
$$

vanishes identically on $\mathcal{M}$, then we have the formula

$$
L \varphi(x)=b(x)=\sum_{i=1}^{n}\left(b^{i}(x)-\sum_{j=1}^{n} \frac{\partial a^{i j}}{\partial x_{j}}(x)\right) \nu_{i} \quad \text { on } \mathcal{M} .
$$

Proof Given each point $x_{0} \in \mathcal{M}$, we choose a local coordinate system

$$
x=\left(x_{1}, \ldots, x_{n-1}, x_{n}\right)
$$

in such a way that

$$
\begin{aligned}
& x_{0}=0, \\
& \varphi(x)=x_{n} \quad \text { near the point } x_{0}, \\
& \boldsymbol{\nu}=(0, \ldots, 0,1) .
\end{aligned}
$$

In terms of this local coordinate system, it suffices to show that

$$
\sum_{j=1}^{n} \frac{\partial a^{n j}}{\partial x_{j}}(0)=0
$$

Indeed, we then have the formula

$$
\begin{aligned}
L \varphi\left(x_{0}\right) & =L \varphi(0)=\sum_{i=1}^{n} a^{i j}(0) \frac{\partial^{2} \varphi}{\partial x_{i} \partial x_{j}}(0)+\sum_{k=1}^{n} b^{k}(0) \frac{\partial \varphi}{\partial x_{k}}(0)=b^{n}(0) \\
& =b^{n}(0)-\sum_{j=1}^{n} \frac{\partial a^{n j}}{\partial x_{j}}(0)=b(0) \\
& =b\left(x_{0}\right) .
\end{aligned}
$$

This proves the desired assertion (4.4).

First, since $0 \in \mathcal{M}$, it follows that

$$
a^{n n}\left(x^{\prime}, 0\right)=0 \quad \text { for all } x^{\prime}=\left(x_{1}, \ldots, x_{n-1}\right) \text { near } 0 \in \mathbf{R}^{n-1} .
$$

Hence, by applying Oleĭnik-Radkevič [21, Corollary 1 to Lemma 1.7.7] we obtain that

$$
\left|a^{n j}\left(x^{\prime}, 0\right)\right|^{2}
$$

$\leq 2 a^{n n}\left(x^{\prime}, 0\right) a^{j j}\left(x^{\prime}, 0\right)$ for all $x^{\prime}$ near the origin in $\mathbf{R}^{n-1}$ if $1 \leq j \leq n-1$, 
so that

$$
a^{n j}\left(x^{\prime}, 0\right)=0 \text { for all } x^{\prime} \text { near the origin in } \mathbf{R}^{n-1} \text { if } 1 \leq j \leq n-1
$$

This proves that

$$
\sum_{j=1}^{n-1} \frac{\partial a^{n j}}{\partial x_{j}}(0)=0
$$

On the other hand, since we have the assertion

$$
a^{n n}\left(x^{\prime}, x_{n}\right) \geq 0 \text { for all } x=\left(x^{\prime}, x_{n}\right) \text { near } 0 \in \mathbf{R}^{n},
$$

it follows from assertion (4.6) that

$$
\frac{\partial a^{n n}}{\partial x_{n}}(0)=0
$$

Therefore, the desired assertion (4.5) follows immediately from assertions (4.7) and (4.8).

The proof of Claim 4.1 is complete.

Step 2: By virtue of formula (4.4), it follows from part (i) of Lemma 4.1 that the assumption (A) implies that the maximum starting at $\mathcal{N}$ goes out of $\mathcal{N}$. Furthermore, we obtain from Lemma 4.1 that if $x_{0}$ is a point of $\mathcal{M}$ such that either

$$
\sum_{i, j=1}^{n} a^{i j}\left(x_{0}\right) \nu_{i} \nu_{j}>0
$$

or

$$
\sum_{i, j=1}^{n} a^{i j}\left(x_{0}\right) \nu_{i} \nu_{j}=0 \quad \text { and } \quad L \varphi\left(x_{0}\right) \neq 0
$$

then the maximum starting at $x_{0}$ either goes into both sides of $\mathcal{M}$ or goes into one side of $\mathcal{M}$, respectively.

Summing up, we obtain that the maximum starting at $\mathcal{M}$ goes out of $\mathcal{M}$.

On the other hand, it follows from part (ii) of Lemma 4.1 that the condition (C) implies that the maximum can traverse $\mathcal{M}$ from every side of $\mathcal{M}$.

Therefore, we can conclude that, under the assumption (A), the maximum at any point $x_{0}$ of $\Omega$ can be propagated into the whole $\Omega$ if and only if one of the conditions (B) and (C) holds true (see [27, Theorem 4.1]).

The proof of Theorem 4.1 is complete.

\section{Necessary conditions}

The conditions (B) and (C) are partially necessary. In fact, we can prove the following second main result: 
Theorem 5.1 (Necessity) Assume that the exceptional set $\mathcal{M}$ is a closed connected hypersurface such that the complement $\Omega \backslash \mathcal{M}$ has two connected components and that its one component $G$ is relatively compact in $\Omega$. Let

$$
\boldsymbol{\nu}=\left(\nu_{1}, \nu_{2}, \ldots, \nu_{n}\right)
$$

be the inward normal to the domain $G$ at the boundary portion $\mathcal{M}$. Assume that

$$
\sum_{i, j=1}^{n} a^{i j}\left(x^{\prime}\right) \nu_{i} \nu_{j} \equiv 0 \quad \text { on } \mathcal{M}
$$

and that

$$
b\left(x^{\prime}\right)=\sum_{i=1}^{n}\left(b^{i}\left(x^{\prime}\right)-\sum_{j=1}^{n} \frac{\partial a^{i j}}{\partial x_{j}}\left(x^{\prime}\right)\right) \nu_{i} \neq 0 \quad \text { on } \mathcal{M} .
$$

Then the strong maximum principle (SMP) does not hold true for the operator L. More precisely, we can construct a function $u^{0} \in C^{\infty}(\Omega)$ such that

$$
\begin{cases}u^{0} \leq 0 & \text { in } \Omega, \\ L u^{0} \geq 0 & \text { in } \Omega, \\ u^{0}=0 & \text { on } \Omega \backslash G, \\ u^{0}\left(x_{0}\right)<0 & \text { for some point } x_{0} \in G .\end{cases}
$$

Remark 5.1 Some remarks are in order.

$1^{\circ}$ Condition (A) holds true, but conditions (B) and (C) do not hold true. Indeed, it suffices to note that

$$
\sum_{i, j=1}^{n} a^{i j}\left(x^{\prime}\right) \nu_{i} \nu_{j}+b\left(x^{\prime}\right)=b\left(x^{\prime}\right) \neq 0 \quad \text { on } \mathcal{M} .
$$

$2^{\circ}$ The assertions (5.3) imply that the maximum zero cannot go into $G$ from the outside $\Omega \backslash G$.

Theorem 5.1 will be proved in the next subsection, due to its length.

We give two simple examples for Theorem 5.1 in the plane $\mathbf{R}^{2}(n=2)$ :

Example 5.1 Let $\Omega=\left\{\left(x_{1}, x_{2}\right):-1<x_{1}<1,-1<x_{2}<1\right\}$ (the rectangle). We let

$$
L_{ \pm}=\exp \left[-\frac{1}{x_{1}^{2}}\right] \frac{\partial^{2}}{\partial x_{1}^{2}}+\frac{\partial^{2}}{\partial x_{2}^{2}} \pm \frac{\partial}{\partial x_{1}} .
$$

Then we have the assertions

$$
\begin{aligned}
& \mathcal{M}=\left\{\left(0, x_{2}\right):-1<x_{2}<1\right\}=\{0\} \times(-1,1) \\
& \mathcal{N}=\emptyset \\
& G_{+}=\left\{\left(x_{1}, x_{2}\right):-1<x_{1}<0,-1<x_{2}<1\right\}=(-1,0) \times(-1,1), \\
& G_{-}=\left\{\left(x_{1}, x_{2}\right): 0<x_{1}<1,-1<x_{2}<1\right\}=(0,1) \times(-1,1) .
\end{aligned}
$$

It is easy to see that the conditions (5.1) and (5.2) hold true for the operators $L_{ \pm}$. Indeed, it suffices to note that

$$
\sum_{i, j=1}^{2} a^{i j}\left(x_{1}, x_{2}\right) \nu_{i} \nu_{j}+b\left(x_{1}, x_{2}\right)= \pm 1 \neq 0 \quad \text { on } \mathcal{M}
$$




\subsection{Proof of Theorem 5.1}

We only consider the case where

$$
b\left(x^{\prime}\right)=\sum_{i=1}^{n}\left(b^{i}\left(x^{\prime}\right)-\sum_{j=1}^{n} \frac{\partial a^{i j}}{\partial x_{j}}\left(x^{\prime}\right)\right) \nu_{i}<0 \quad \text { on } \mathcal{M},
$$

since the case where $b\left(x^{\prime}\right)>0$ on $\mathcal{M}$ can be treated in a similar way. The proof of Theorem 5.1 is divided into three steps.

Step 1: First, we choose a function $\psi \in C_{0}^{\infty}(\Omega)$ such that

$$
\begin{cases}\psi \leq 0 & \text { in } G \\ \psi\left(x_{0}\right)=-1 & \text { for some point } x_{0} \in G\end{cases}
$$

Then it follows from an application of Stroock-Varadhan [27, Corollary 8.2] that there exists a unique solution $u \in L^{\infty}(G)$ of the Dirichlet problem

$$
\begin{cases}L u=-\psi & \text { in } G \\ u=0 & \text { on } \partial G\end{cases}
$$

and further that it is given by the following probabilistic representation formula:

$$
u(x)=E_{x}\left[\int_{0}^{\tau^{\prime}} \psi(\xi(t)) \exp \left(\int_{0}^{t} c(\xi(s)) d s\right) d t\right] .
$$

Here:

(1) $\xi(t)=\left(\xi^{1}(t), \xi^{2}(t), \ldots, \xi^{n}(t)\right)$ is a diffusion process governed by the system of stochastic differential equations

$$
d \xi^{i}(t)=\sqrt{2} \sum_{j=1}^{n} \sigma^{i j}(\xi(t)) \circ d B^{j}(t)+\left(b^{i}(\xi(t))-\sum_{j=1}^{n} \frac{\partial a^{i j}}{\partial x_{j}}(\xi(t))\right) d t
$$

where $\sigma=\left(\sigma^{i j}\right)$ is a unique square root of the non-negative definite matrix $\left(a^{i j}\right), B(t)=\left(B^{1}(t), B^{2}(t), \ldots, B^{n}(t)\right)$ is an $n$-dimensional standard Brownian motion and the stochastic integral is defined in the Fisk-Stratonovich sense (cf. [25]).

(2) $\tau^{\prime}$ is the exit time from the compact set $\bar{G}=G \cup \mathcal{M}$ defined by the formula

$$
\tau^{\prime}=\inf \{t \geq 0: \xi(t) \notin \bar{G}\} .
$$

Indeed, since the Lie algebra $\mathcal{L}\left(X_{1}, X_{2}, \ldots, X_{n}\right)$ has rank $n$ at each point of $G$ and since we have, by condition (5.2),

$$
b\left(x^{\prime}\right)=\sum_{i=1}^{n}\left(b^{i}\left(x^{\prime}\right)-\sum_{j=1}^{n} \frac{\partial a^{i j}}{\partial x_{j}}\left(x^{\prime}\right)\right) \nu_{i}<0 \quad \text { on } \mathcal{M},
$$

it is easily seen from Chow's theorem [5, Satz C] and Stroock-Varadhan [27, Remark 5.2] that

$$
\sup _{x \in \bar{G}} E_{x}\left[\tau^{\prime}\right]<\infty .
$$


It should be emphasized that the probabilistic formula (5.6) can be traced back to the pioneering work of Kakutani [17] on harmonic functions.

Therefore, our desired assertion follows from an application of [27, Corollary 8.2] with

$$
\begin{aligned}
& \Sigma_{2}:=\left\{x^{\prime} \in \partial G: \sum_{i, j=1}^{n} a^{i j}\left(x^{\prime}\right) \nu_{i} \nu_{j}=0, b\left(x^{\prime}\right)<0\right\}=\mathcal{M}, \\
& \Sigma_{3}:=\partial G \backslash \mathcal{M} .
\end{aligned}
$$

Step 2: Secondly, since the function $\psi(x)$ satisfies the conditions (5.5), we obtain from the probabilistic representation formula (5.6) that

$$
\left\{\begin{array}{l}
u^{0} \leq 0 \text { in } G \\
u^{0}\left(x_{0}\right)<0 \text { for some point } x_{0} \in G .
\end{array}\right.
$$

Now we let

$$
u^{0}(x)= \begin{cases}u(x) & \text { for } x \in G, \\ 0 & \text { for } x \in \Omega \backslash G,\end{cases}
$$

and

$$
(L u)^{0}(x)= \begin{cases}L u(x) & \text { for } x \in G, \\ 0 & \text { for } x \in \Omega \backslash G .\end{cases}
$$

By virtue of condition (5.1), in a tubular neighborhood $V$ of $\mathcal{M}$ we can express the operator $L$ in the form

$$
L=a(x) \frac{\partial^{2}}{\partial \nu^{2}}+\ell_{1}\left(x, D^{\prime}\right) \frac{\partial}{\partial \nu}+\ell_{2}\left(x, D^{\prime}\right) .
$$

Here

(1) $\partial / \partial \nu$ denotes the inward normal differentiation to the domain $G$ at the boundary portion $\mathcal{M}$.

(2) $\ell_{j}\left(x, D^{\prime}\right), j=1,2$, is a differential operator of order $j$ acting along the parallel surfaces of $\mathcal{M}$ on $V$.

(3) $a(x)=\frac{\partial a}{\partial \nu}(x)=0$ on $\mathcal{M}$, since $a(x) \geq 0$ on $V$.

Therefore, by using the well-known jump formula (see [4, Chapitre 1, formule (1.4.1)]; [13, formula (2.2.1)]) we obtain that

$$
L\left(u^{0}\right)=(L u)^{0}+a(x) \frac{\partial}{\partial \nu}\left(u \delta_{\mathcal{M}}\right)+\ell_{1}\left(x, D^{\prime}\right)\left(u \delta_{\mathcal{M}}\right),
$$

where $\delta_{\mathcal{M}}$ is the surface measure on the hypersurface $\mathcal{M}$ defined by the formula

$$
\left\langle\delta_{\mathcal{M}}, \theta\right\rangle=\left.\int_{\mathcal{M}} \theta\right|_{\mathcal{M}} d \sigma \quad \text { for all } \theta \in C_{0}^{\infty}(V)
$$

and $d \sigma$ is the surface element on $\mathcal{M}$.

However, since $a(x)=\frac{\partial a}{\partial \nu}(x)=0$ and $u(x)=0$ on $\mathcal{M}$, it follows from formula (5.8) and assertion (5.5) that

$$
L\left(u^{0}\right)=(L u)^{0}=(-\psi)^{0}=-\psi \geq 0 \quad \text { in } \Omega .
$$


Step 3: Finally, by assertions (5.7) and (5.9) it remains to show that $u^{0} \in$ $C^{\infty}(\Omega)$.

To do so, we recall that a differential operator $A$ with $C^{\infty}$ coefficients in $\Omega$ is said to be hypoelliptic if it satisfies the condition

$$
\operatorname{sing} \operatorname{supp} u=\operatorname{sing} \operatorname{supp} A u \quad \text { for every distribution } u \in \mathcal{D}^{\prime}(\Omega) .
$$

It is easy to see that this condition is equivalent to the following: For any open subset $\Omega_{1}$ of $\Omega$, we have the assertion

$$
u \in \mathcal{D}^{\prime}(\Omega), A u \in C^{\infty}\left(\Omega_{1}\right) \Longrightarrow u \in C^{\infty}\left(\Omega_{1}\right) .
$$

For example, it is known (see [4], [16], [21]) that all elliptic operators are hypoelliptic. Moreover, we say that $A$ is globally hypoelliptic if it satisfies the weaker condition

$$
u \in \mathcal{D}^{\prime}(\Omega), A u \in C^{\infty}(\Omega) \Longrightarrow u \in C^{\infty}(\Omega) .
$$

It should be noticed that these two notions may be transferred to manifolds.

Since we have, by assumption (3.1), conditions (5.1) and (5.4),

$$
\begin{aligned}
& \mathcal{M}=\{x \in \Omega: \varphi(x)=0\} \\
& \boldsymbol{\nu}=\operatorname{grad} \varphi \neq 0 \\
& \sum_{i, j=1}^{n} a^{i j}(x) \nu_{i} \nu_{j} \equiv 0 \quad \text { on } \mathcal{M}, \\
& b\left(x^{\prime}\right)=L \varphi\left(x^{\prime}\right)<0 \quad \text { on } \mathcal{M},
\end{aligned}
$$

it follows from an application of Oleĭnik-Radkevič [21, Theorem 2.6.3] (see also [23, Theorem 7] and [15, Theorem 5.9]) that the operator $L$ is globally hypoelliptic in $\Omega$.

Hence, we obtain from equation (5.9) that

$$
u^{0} \in C^{\infty}(\Omega)
$$

since $\psi \in C_{0}^{\infty}(\Omega)$.

Summing up, we have constructed a function $u^{0} \in C^{\infty}(\Omega)$ which satisfies the desired conditions (5.3).

Now the proof of Theorem 5.1 is complete.

\section{Propagation of maximums in terms of subunit vectors}

In this section, we formulate another maximum principle for the differential operator

$$
L u=\sum_{i, j=1}^{n} a^{i j}(x) \frac{\partial^{2} u}{\partial x_{i} \partial x_{j}}+\sum_{i=1}^{n} b^{i}(x) \frac{\partial u}{\partial x_{i}}+c(x) u
$$

in terms of subunit vectors introduced by Fefferman-Phong [6]. This section is adapted from [31, Chapter 7].

Now we consider the following problem: 
Problem 6.1 Let $x$ be a point of $\Omega$. Determine the largest connected, relatively closed subset $D(x)$ of $\Omega$, containing $x$, such that

$$
\left\{\begin{array}{l}
\text { If } u \in C^{2}(\Omega), L u \geq 0 \text { in } \Omega \text { and if } u \text { takes its greatest non-negative } \\
\text { value } M \text { at } x, \text { then } u \equiv M \text { throughout } D(x) .
\end{array}\right.
$$

The set $D(x)$ is called the propagation set of $x$ in $\Omega$.

We shall give a coordinate-free description of the set $D(x)$ in terms of subunit vectors. A tangent vector

$$
X=\sum_{j=1}^{n} \gamma^{j} \frac{\partial}{\partial x_{j}} \in T_{x}(\Omega)
$$

at $x \in \Omega$ is said to be subunit for the differential operator $L$ if it satisfies the condition

$$
\left(\sum_{j=1}^{n} \gamma^{j} \eta_{j}\right)^{2} \leq \sum_{i, j=1}^{n} a^{i j}(x) \eta_{i} \eta_{j} \quad \text { for all } \eta=\sum_{j=1}^{n} \eta_{j} d x_{j} \in T_{x}^{*}(\Omega),
$$

Since this notion is coordinate-free, we rotate the coordinate axes so that the symmetric matrix $\left(a^{i j}\right)$ is diagonalized at $x$ :

$$
\left(a^{i j}(x)\right)=\left(\lambda_{i} \delta_{i j}\right), \quad \lambda_{1}>0, \ldots, \lambda_{r}>0, \quad \lambda_{r+1}=\ldots=\lambda_{n}=0
$$

where $r=\operatorname{rank}\left(a^{i j}(x)\right)$. Then it is easy to see that the tangent vector $X$ is subunit for $L$ if and only if it is contained in the following ellipsoid of dimension $r$ :

$$
\frac{\left(\gamma^{1}\right)^{2}}{\lambda_{1}}+\ldots+\frac{\left(\gamma^{r}\right)^{2}}{\lambda_{r}} \leq 1, \quad \gamma^{r+1}=\ldots=\gamma^{n}=0 .
$$

A subunit trajectory is a Lipschitz path $\gamma:\left[t_{1}, t_{2}\right] \rightarrow \Omega$ such that the tangent vector

$$
\dot{\gamma}(t)=\frac{d}{d t}(\gamma(t))
$$

is subunit for $L$ at $\gamma(t)$ for almost every $t$. We remark that if $\dot{\gamma}(t)$ is subunit for $L$, so is $-\dot{\gamma}(t)$; hence subunit trajectories are not oriented.

We let

$$
X_{0}(x)=\sum_{i=1}^{n}\left(b^{i}(x)-\sum_{j=1}^{n} \frac{\partial a^{i j}}{\partial x_{j}}(x)\right) \frac{\partial}{\partial x_{i}} .
$$

The vector field $X_{0}(x)$ is called the drift vector field in probability theory, while it is the so-called subprincipal part of the operator $L$ in terms of the theory of partial differential equations (see [16, formula (1.8.32)]).

A drift trajectory is a curve $\theta:\left[t_{1}, t_{2}\right] \rightarrow \Omega$ such that

$$
\dot{\theta}(t)=X_{0}(\theta(t)) \quad \text { on }\left[t_{1}, t_{2}\right],
$$

and this curve is oriented in the direction of increasing $t$.

Our main result reads as follows (see [31, Theorem 7.2.1]):

The propagation set $D(x)$ of $x$ in $\Omega$ contains the closure $D^{\prime}(x)$ in $\Omega$ of 
all points $y \in \Omega$ which can be joined to $x$ by a finite number of subunit and drift trajectories.

This result (PMP) is called the sharp maximum principle for the differential operator $L$ (cf. [11], [24]). The sharp maximum principle says that if the matrix $\left(a^{i j}(x)\right)$ is non-degenerate at a point $x$, that is, if $r=\operatorname{rank}\left(a^{i j}(x)\right)=n$, then the maximum propagates in an open neighborhood of $x$; but if the matrix $\left(a^{i j}(x)\right)$ is degenerate at $x$, then the maximum propagates only in a "thin" ellipsoid of dimension $r$ (cf. formula (6.2)) and in the direction of $X_{0}$. Now we see the reason why the strong maximum principle (1.1) holds true for the Laplacian $\Delta$.

We give four simple examples of the strong maximum principle (SMP) via the sharp maximum principle (PMP) in the case where $\Omega$ is the square $(-1,1) \times(-1,1)$ in the plane $\mathbf{R}^{2}$ :

Example 6.1 $L_{1}=\partial^{2} / \partial x_{1}^{2}+x_{1}^{2} \partial^{2} / \partial x_{2}^{2}$. The subunit vector fields for $L_{1}$ are generated by the following:

$$
\left(\frac{\partial}{\partial x_{1}}, x_{1} \frac{\partial}{\partial x_{2}}\right)
$$

Hence we have the assertion

$$
\text { The set } D^{\prime}\left(\left(x_{1}, x_{2}\right)\right) \text { is equal to } \Omega \text { for every }\left(x_{1}, x_{2}\right) \in \Omega \text {. }
$$

Namely, the strong maximum principle (SMP) holds true for the operator $L_{1}$, as is shown in Example 1.1.

Example 6.2 $L_{4}=x_{1}^{2} \partial^{2} / \partial x_{1}^{2}+\partial^{2} / \partial x_{2}^{2}$. The subunit vector fields for $L_{4}$ are generated by the following:

$$
\left(x_{1} \frac{\partial}{\partial x_{1}}, \frac{\partial}{\partial x_{2}}\right)
$$

Thus we have the assertion

$$
D^{\prime}\left(\left(x_{1}, x_{2}\right)\right)= \begin{cases}{[0,1) \times(-1,1)} & \text { if } x_{1}>0 \\ \{0\} \times(-1,1) & \text { if } x_{1}=0 \\ (-1,0] \times(-1,1) & \text { if } x_{1}<0\end{cases}
$$

It can be shown that the strong maximum principle (SMP) does not hold true for the operator $L_{4}$, just as in Example 5.1.

Example $6.3 L_{5}=x_{1}^{2} \partial^{2} / \partial x_{1}^{2}+\partial^{2} / \partial x_{2}^{2}+x_{2} \partial / \partial x_{1}$. The subunit vector fields for $L_{5}$ are generated by formula (6.4), and the drift vector field is equal to the following:

$$
X_{0}\left(x_{1}, x_{2}\right)=\left(x_{2}-2 x_{1}\right) \frac{\partial}{\partial x_{1}} .
$$

Thus, by virtue of the drift vector field we have assertion (6.3), and so the strong maximum principle (SMP) holds true for the operator $L_{5}$. We remark that the assumptions (A) and (C) hold true for the operator $L_{5}$, just as in Example 4.2. 
Example 6.4 $L_{6}=x_{1}^{2} \partial^{2} / \partial x_{1}^{2}+\partial^{2} / \partial x_{2}^{2}+\partial / \partial x_{1}$. The subunit vector fields for $L_{6}$ are generated by the vector fields (6.4), and the drift vector field is equal to the following:

$$
X_{0}\left(x_{1}, x_{2}\right)=\left(1-x_{1}\right) \frac{\partial}{\partial x_{1}} .
$$

Hence we have the assertion

$$
D^{\prime}\left(\left(x_{1}, x_{2}\right)\right)= \begin{cases}\Omega & \text { if } x_{1}<0 \\ {[0,1) \times(-1,1)} & \text { if } x_{1} \geq 0\end{cases}
$$

It can be shown that the strong maximum principle (SMP) does not hold true for the operator $L_{6}$ in some weak sense, just as in Example 5.1.

\section{Concluding remarks}

Some remarks are in order.

(1) The maximum principles in this paper are adapted from Amano [1], Bony [2], Gilbarg-Trudinger [10], Hill [11], Oleŭnik [20], Oleĭnik-Radkevič [21] and Redheffer [24]. For a general study of maximum principles, the reader might refer to Bony-Courrège-Priouret [3], Protter-Weinberger [22] and also [32, Chapter 8].

(2) Our formulation of the strong maximum principle is coordinate-free. The results here may be applied to questions of uniqueness for degenerate elliptic boundary value problems on a manifold.

(3) The underlying analytical mechanism of propagation of maximums plays an important role in the probabilistic interpretation and study of Markov processes from the viewpoint of functional analysis (see Bony-Courrège-Priouret [3, Chapitre I], [29] and [32, Part III]).

(4) The propagation set $D(x)$ coincides with the support of the Markov process corresponding to the operator $L$, which is the closure of the collection of all possible trajectories of a Markovian particle, starting at $x$, with generator $L$ (see Stroock-Varadhan [26, Theorem 5.2]).

(5) It seems quite likely that there is an intimate connection between propagation of maximums and propagation of singularities for degenerate elliptic differential operators of second-order (see Fujiwara-Omori [9], Hörmander [14], Yoshino [33] and [30]).

Acknowledgements The author is grateful to the referee for many valuable suggestions and comments, which have improved substantially the presentation of the present paper. He is also indebted to Professors Hajime Sato and Koichi Uchiyama for formulating the mapping $\Psi$ in terms of differential geometry.

\section{References}

1. Amano, K.: Maximum principles for degenerate elliptic-parabolic operators. Indiana Univ. Math. J. 29, 545-557 (1979)

2. Bony, J.-M.: Principe du maximum, inégalité de Harnack et unicité du problème de Cauchy pour les opérateurs elliptiques dégénérés, Ann. Inst. Fourier (Grenoble), 19, 277-304 (1969) 
3. Bony, J.-M., Courrège. P., Priouret, P.: Semigroupes de Feller sur une variété à bord compacte et problèmes aux limites intégro-différentiels du second ordre donnant lieu au principe du maximum, Ann. Inst. Fourier (Grenoble), 18, 369-521 (1968)

4. Chazarain, J., Piriou, A.: Introduction à la théorie des équations aux dérivées partielles linéaires, Gauthier-Villars, Paris (1981)

5. Chow, W.-L.: Über Systeme von linearen partiellen Differentialgleichungen erster Ordnung, Math. Ann. 117, 98-105 (1940)

6. Fefferman, C., Phong, D. H.: Subelliptic eigenvalue problems, In: Conference on Harmonic analysis (1981: Chicago, Ill), 590-606, Wadsworth, Belmont, California (1983)

7. Fichera, G.: Sulle equazioni differenziali lineari ellittico-paraboliche del secondo ordine, Atti Accad. Naz. Lincei. Mem. Cl. Sci. Fis. Mat. Nat. Sez. I. (8) 5, 1-30 (1956)

8. Friedman, A.: Remarks on the maximum principle for parabolic equations and its applications. Pacific J. Math. 8, 201-211 (1958)

9. Fujiwara, D.,Omori, H.: An example of a globally hypo-elliptic operator, Hokkaido Math. J. 12, 293-297 (1983)

10. Gilbarg, D., Trudinger, N. S.: Elliptic partial differential equations of second order, Reprint of the 1998 edition, Classics in Mathematics, Springer-Verlag, Berlin (2001)

11. Hill, C. D.: A sharp maximum principle for degenerate elliptic-parabolic equations. Indiana Univ. Math. J. 20, 213-229 (1970)

12. Hopf, E.: Elementare Bemerkungen über die Lösungen partieller Differentialgleichungen zweiter ordnung vom elliptischen Typus, Sitz. Ber. Preuss. Akad. Wissensch. Berlin Math.Phys. Kl. 19, 147-152 (1927)

13. Hörmander, L.: Pseudodifferential operators and non-elliptic boundary problems. Ann. of Math. (2), 83, 129-209 (1966)

14. Hörmander, L.: Hypoelliptic second order differential equations. Acta Math. 119, 147-171 (1967)

15. Hörmander, L.: A class of hypoeiliptic pseudodifferential operators with double characteristics. Math. Ann. 217, 165-188 (1975)

16. Hörmander, L.: The analysis of linear partial differential operators III, Pseudo-differential operators, reprint of the 1994 edition, Grundlehren der mathematischen Wissenschaften, Springer-Verlag, Berlin Heidelberg New York Tokyo (2007)

17. Kakutani, S.: Two-dimensional Brownian motion and harmonic functions. Proc. Imp. Acad. 20, 706-714 (1944)

18. Nicolaescu, L. I.: Lectures on the geometry of manifolds, second edition, World Scientific Publishing, Hackensack, New Jersey (2007)

19. Nirenberg, L.: A strong maximum principle for parabolic equations. Comm. Pure Appl. Math. 6, 167-177 (1953)

20. Oleĭnik, O. A.: On properties of solutions of certain boundary problems for equations of elliptic type. Mat. Sbornik 30, 595-702 (1952) (in Russian).

21. Oleı̆nik, O. A., Radkevič, E. V.: Second order equations with nonnegative characteristic form, Itogi Nauki, Moscow (1971) (in Russian); English translation: Amer. Math. Soc., Providence, Rhode Island and Plenum Press, New York (1973)

22. Protter, M. H., Weinberger, H. F.: Maximum principles in differential equations, corrected second printing, Springer-Verlag, New York (1984)

23. Radkevič, E. V.: A priori estimates and hypoelliptic operators with multiple characteristics (in Russian). Dokl. Akad. Nauk SSSR 187, 274-277 (1969): English translation in Soviet Math. Dokl. 10, 849-853 (1969)

24. Redheffer, R. M.: The sharp maximum principle for nonlinear inequalities, Indiana Univ. Math. J. 21, 227-248 (1971)

25. Stratonovich, R. L.: A new representation for stochastic integrals and equations. SIAM J. Control 4, 362-371 (1966)

26. Stroock, D. W., Varadhan, S. R. S.: On the support of diffusion processes with applications to the strong maximum principle. In: Proceedings of the Sixth Berkeley Symposium of Probability and Mathematical Statistics, Vol. III, 333-359 (1972)

27. Stroock, D. W., Varadhan, S. R. S.: On degenerate elliptic-parabolic operators of second order and their associated diffusions. Comm. Pure Appl. Math. 25, 651-713 (1972)

28. Taira, K.: A strong maximum principle for degenerate elliptic operators. Comm. Partial Differential Equations 4, 1201-1212 (1979)

29. Taira, K.: Semigroups and boundary value problems. Duke Math. J. 49, 287-320 (1982)

30. Taira, K.: Le principe du maximum et l'hypoellipticité globale. Séminaire Bony-SjöstrandMeyer 1984-1985, Exposé No. I, Ecole Polytechnique, Palaiseau (1985) 
31. Taira, K.: Diffusion processes and partial differential equations, Academic Press Inc., Boston, Massachusetts (1988)

32. Taira, K.: Semigroups, boundary value problems and Markov processes, second edition, Springer Monographs in Mathematics. Springer-Verlag, Heidelberg (2014)

33. Yoshino, M.: A class of globally hypoelliptic operators on the torus. Math. Z. 201, 1-11 (1989) 